\title{
Exploring subjective well-being in social network sites? - Supplementary fit and social support perspective
}

\author{
Ying Chieh Liu ${ }^{1}$, Hung Yi Chen ${ }^{1 a}$, and Bo Yen Yao ${ }^{1}$ \\ ${ }^{1}$ Chaoyang University of Technology, 168, Jifeng E. Rd., Wufeng District, Taichung, 41349, Taiwan
}

\begin{abstract}
This study formed a framework theorized on supplementary fit and social support to explore users' perceptions of Subject Well-Being in Social Network Sites. Online surveys were conducted in Facebook and Google+. Empirical results showed that value-based fit and personal-based fit promoted the obtaining of emotional and informational support but not instrumental support, and emotional and informational support had significant impacts on life satisfaction and happiness while instrumental support had no significant impacts on life satisfaction and happiness. Our model pinpointed novel ideas to present how individuals obtain social supports through Social Network Sites and how these social supports influence happiness and life satisfaction.
\end{abstract}

\section{Introduction}

Recent research has proposed many factors affecting SWB (Subjective Well-Being) and empirically examined them, such as socializing, self-esteem, loneliness, selfdisclosure, social integration, social bonding, social bridging [1, 2]. However, little research has studied SWB in the SNS (Social Network Sites) in the viewpoint of social support. The traditional application of social support focuses on the linkages between health treatment and supports provided by people around patients ex., [3, 4]. But our study focuses on ordinary people who interact with their friends in SNS instead of patients with specific health issue. Additionally, little research has explored the antecedents influencing the obtaining of social support. We applied supplementary fit to fill in this gap. Supplementary fit refers to the similar or matching characteristics between a person and other group members [5]. It occurs when "a person supplements, embellishes, or possesses characteristics which are similar to other individuals in the environment" [6]. From this point of view, we assume that individuals perceive social supports when supplementary fit occurs between individuals and the other members in SNS. Thus, the purpose of this study is to: (a) Identify how supplementary fit affects people's perception of social support in the context of SNS; (b) Explore how people's perception of social support influences SWB in the context of SNS.

\section{Literature review and hypotheses development}

\footnotetext{
a Corresponding author: hychen39@gmail.com
}

In the context of SNS, individuals interact through website platform to obtain emotional or practical support to satisfy their needs and improve their ability to adapt the environment. People around the world can be in individuals' social support sys-tem, as long as they are using the same SNS. We synthesize social support as emotional, instrumental and informational support. Emotional support refers to demonstrations of love and caring, esteem and value, encouragement, and sympathy. Instrumental support consists of offering or supplying behavioral or material assistance with practical tasks or problems, such as goods and services. Informational support is the provision of facts or advice that may help a person solve problems.

According to the similarity theory, people are more attracted to those who are similar to them in aspects like demographic background, personality trait, values and socio-economic background. In a relationship consisting of like-minded individuals, sharing the same viewpoints allows them to feel that they are accepted by others, and thereby their own confidence increases and the relationship reinforces. Supplementary fit, which is applied in this study, can be categorized into value-based fit and personality-based fit [7]. The former one emphasizes on the match of value and the latter one focuses on math of personality traits between individuals and other group members. In a context of SNS, valuebased fit regards to value congruence between individuals and the SNS. Individuals use values to make choices of the extent they participate in the SNS activities. The SNS members use values to develop a norm to guide members' beliefs and behaviors. The match between the individual's values and the SNS values can facilitate the interactions between individual and other members, and 
further improve harmony of the SNS and intentions providing supports for each other. Thus, we assume that value-based fit promotes more exchange of social support and the three hypotheses are described below.

H1a: Value-based fit promotes the obtaining of emotional support for individuals.

H1b:Value-based fit promote the obtaining of instrumental support for individuals.

H1c: Value-based fit promote the obtaining of informational support for individuals.

Personality, in this study, instead of specifying to demographic variables (such as race, age, gender) but a stable characteristics and is proximal to behavior. In a context of SNS, personality-based fit implies to the psychological and behavioral compatibility between individuals and other members in SNS. With personalitybased fit occurs, individuals feel more comfortable and are willing to help others. Thus, we assume that personality-based fit promotes more exchange of social support which includes emotional, instrumental and information support. The three hypotheses are described below.

H2a: Personality -based fit can promote the obtaining of emotional support for individuals.

H2b: Personality -based fit can promote the obtaining of instrumental support for individuals.

H2c: Personality -based fit can promote the obtaining of informational support for individuals.

We classified SWB as twofold: life satisfaction and happiness. Life satisfaction is more reflective of the broad and continuing circumstances of an individual's life, while happiness is more volatile. We assume that three kind of social supports (emotional, instrumental and informational support) can improve the outlook of life, letting people more optimistic, lightens the stress of life and further increase life satisfaction and happiness. Thus, the hypotheses are proposed below.

$\mathrm{H} 3 \mathrm{a}$ : Emotional support is positively related to life satisfaction.

$\mathrm{H} 3 \mathrm{~b}$ : Emotional support is positively related to happiness.

$\mathrm{H} 4 \mathrm{a}$ : Instrumental support is positively related to life satisfaction.

H4b: Instrumental support is positively related to happiness.

H5a: Informational support is positively related to life satisfaction.

H5b: Informational support is positively related to happiness.

\section{Research methodology}

To examine the proposed hypotheses, we conducted surveys on Facebook and Google+. My3q (www.my3q.com) was used as our questionnaire distribution platform. The duration lasted for one month and 450 validated questionnaires were collected. Most of the subjects had bachelor degree or higher $(87 \%)$. Females (53\%) were higher than males (47\%). The ages ranged from 20 to 30 accounted for $74 \%$. Subjects reported that $78 \%$ of Facebook or Google+ friends come from people they know in real life.

The overall fit of our structural model was quite satisfactory by CFA $(\chi 2 / \mathrm{df} .=2.42$, $\mathrm{RMSEA}=0.58$, CFI $=0.97, \mathrm{IFI}=0.95$, GFI $=0.91, \mathrm{AGFI}=0.88)$. SEM (Structural Equation Modeling) then was applied to estimate the path diagram of the framework in the method of maximum likelihood. Figure 1 shows the path diagram. As seen in Figure 2, there are four insignificant paths (value-based fit, personality-based fit • instrumental support, Instrumental support • Life satisfaction, Happiness). The hypothesis test was engaged according to the path diagram. Thus, the Hypothesis 1a, $1 c, 2 a, 2 c, 3 a, 3 b, 5 a$ and $5 b$ were supported while the Hypothesis 1b, 2b, 4a and $4 b$ were not.

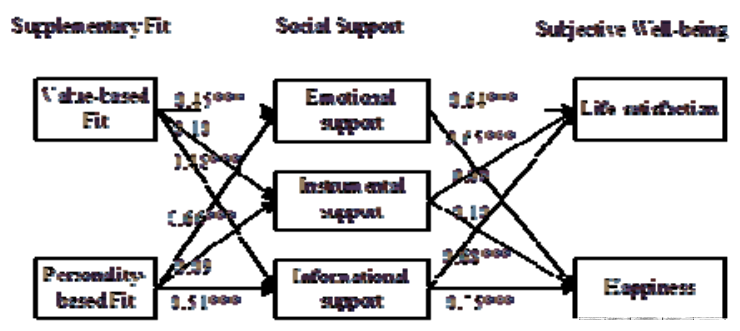

Figure 1. The result of structural equation model

\section{Discussions}

The hypothesis 1a and 1c were supported but not $1 \mathrm{~b}$, which proves that value-based fit promotes emotional and informational support but not instrumental support. That is to say, individuals perceive more emotional and informational support when the match between the individual's values and other SNS members' values occurs. Also, the hypothesis $2 \mathrm{a}$ and $2 \mathrm{c}$ were supported but not $2 b$, which proves that personality-based fit promotes emotional and informational support but not instrumental support. That is to say, individuals perceive more emotional and in-formational support when the match between the individual's personality traits and other SNS members' personality traits occurs. These results give us two implications. First, emotional and information support are provided when value-based fit and personality-based fit occur. Second, instrumental sup-port seems to be difficult to appear even value-based fit and personality-based fit exists.

By the significant support of the hypothesis $3 a, 3 b, 5 a$ and $5 \mathrm{~b}$, both emotional support and informational support had significant impacts on life satisfaction and happiness, which is correspondent to the past studies. It can be inferred that emotional and in-formational support can be a buffer to people's stress and a solution resource to solve the issues in people's lives. For example, when users post their job frustration and express bad emotions, their friends may comfort them by showing sympathy or even providing some tips to solve the issues. This would improve users' degree of happiness and life satisfaction. These results also indicate that SNS has the same 
functions as traditional interpersonal relationship network in term of providing emotion and information support to help people be happier and satisfy with their lives.

However, the insignificant support of hypothesis $3 \mathrm{a}$ and $3 \mathrm{~b}$ indicated that instrumental support had no significant impacts on life satisfaction and happiness. The reason could come from the essence of SNS. Instrumental support refers to specific material and practical aids provided by others. In a virtual environment of SNS, online friends can hardly pro-vide immediate and practical aids due to they may not check the messages anytime or they are geo-graphically far from the helpseeker, which leads that users perceive little instrumental support from their online friends. To improve this, SNS managers can develop a "call for help" push function to enable users to transmit their messages immediately to the assigned friends through various communication tools, such as text message, phone call, Line, WhatsApp, WeChat...etc, or even combine GPS (Global Positioning System) to search friends nearby to require their assistance.

To summarize the discussion above, emotional and information support are provided but not instrumental support when value-based fit and personality-based fit occurs in the context of SNS. Individuals gain emotional and information support and improve life satisfaction and happiness when they share their inhibition, pressure, depression and excitement with online friends through SNS. In terms of emotional and information support, SNS can play the same role as interpersonal relationship in real world. Additionally, subjects reported that $78 \%$ of online friends in SNS come from people they know in real life, which means SNS may help users improve intimacy with their friends. That is to say, SNS does not weaken substantial relationships in re-al world; on the contrary, it enhances existing relationships through emotional and information sup-port, and in turn improves users' satisfaction and happiness.

\section{Conclusions}

This study made several contributions. First, we applied value-based fit and personal-based fit as antecedent variables to explain why social support can be obtained. Additionally, the empirical test showed that emotional and information support are available when value-based fit and personality-based fit occur. Second, different from the past studies regarding social support as a singledimensional factor, this study regarded it as a multidimensional factor and conceptualized it as emotional, instrumental and in-formational support, which identifies the supports that people are able to retrieve from SNS. Also, SWB was conceptualized as momentary happiness and long-term life satisfaction. Our model pinpointed novel ideas to present how individuals obtain social supports through SNS and how the three sort of social supports influence users' happiness and life satisfaction. Third, supplementary fit and social sup-port theory were proved well explained people's interactive relationships in SNS.

\section{References}

1. V. Apaolaza, P. Hartmann, E. Medina, J.M. Barrutia, C. Echebarria, Comput. Hum. Behav. 29, 4, 12821289 (2013)

2. K. T. Lee, M. J. Noh, D. M. Koo. Cyberpsychol Behav. Soc. Netw, 16, 6, 413-418 (2013)

3. C. H. Kroenke, M. L. Kwan, A. I. Neugut, I. J. Er-gas, J. D. Wright, B. J. Caan. Breast. Cancer. Res. Treat, 139, 2, 515-527 (2013)

4. F. J. H. Lu, Y. Hsu. J. Athl. Training, 48, 1, 92-98. (2013)

5. D. M. Cable, J. R. Edwards. J. App. Psych, 89, 5 822834 (2004)

6. P. M. Muchinsky, C. J. Monahan. J. Vocat. Behav, 31, 3, 268-277 (1987)

7. J. Y. Seong, A. L. Kristof-Brown. J. Manag. Psych, 27, 6, 536-556 (2012) 